\title{
Reactions related with hydroxyl, carboxyl and alkyl side chain at different temperature stages and the effects on low-rank coal ignition
}

\author{
Shengyue $\mathrm{Ma}^{1}$, Jie Xiong ${ }^{1}$, Jing Xiao ${ }^{1}$, Yueling Zhang ${ }^{1}$, Ruihong Zhang ${ }^{1}$, Yajun Tian ${ }^{2}$, and Kechang Xie ${ }^{3}$ \\ ${ }^{1}$ MOE Key Laboratory of Resources and Environmental System Optimization, School of Environmental Science and Engineering, North \\ China Electric Power University, Beijing, 102206,China \\ ${ }^{2}$ New Energy Research Center, National Institute of Clean-and-Low-Carbon Energy, Beijing, 102211, China \\ ${ }^{3}$ Key Laboratory of Coal Science and Technology of Ministry of Education and Shanxi Province, Institute of Coal Chemical Engineering, \\ Taiyuan University of Technology, Shanxi, 030024,China
}

\begin{abstract}
Low-rank coal contains abundant hydroxyl, carboxyl and alkyl side chains, and reactions related to these groups are the main reason for the spontaneous combustion of low-rank coal. Here, two different low-rank coals (BRXL, YJL52) are selected. Firstly, the ignition temperatures of the coals are determined by thermogravimetric method. Secondly, the coals are heated at $100{ }^{\circ} \mathrm{C}$ temperature intervals before the ignition temperature in the thermogravimetry, and infrared measurement is performed to explore the changes of these groups. Combining previous studies in the literatures with infrared analysis, it is found that reactions related are as follows: phenolic hydroxyl converting into alcoholic hydroxyl, alcoholic hydroxyl further oxidizing to carboxyl, and carboxyl decarboxylating into alkyl side chains. After that, the changes of phenolic hydroxyl and carboxyl on the surface of the coal at $100{ }^{\circ} \mathrm{C}$ temperature intervals are determined by titration, which further reveal the main reactions occurred in every temperature interval. Additionally, the actual heat release in different temperature ranges is discussed with the reaction enthalpies of the above-mentioned main reactions. As a result, the key temperature stage that causes spontaneous combustion is found. The screening study in this paper on the reaction of low-rank coal before spontaneous combustion provides a theoretical basis for the control of spontaneous combustion of low-rank coal.
\end{abstract}

\section{Introduction}

In-depth study of the spontaneous combustion mechanism of low-rank coal can effectively prevent and control its spontaneous combustion [1]. So far, scholars have studied the spontaneous combustion of low-rank coal to determine its spontaneous combustion tendency. For example, the coal-oxygen reaction kinetic simulation method [2] mainly judged the speed of the coal-oxygen reactions from the obtained activation energy value, and the coal with the fast reaction was easier to spontaneous combustion. The critical moisture value determination method [3] was studied from the perspective of the positive and negative effects of different content of moisture in the reaction. The indicator gas method [4] revealed the oxygen reaction of coal before spontaneous combustion by analyzing the gas emitted during low-temperature oxidation of coal. These studies only speculated on the causes of spontaneous combustion of low-rank coal from macroscopic phenomena and could not explain the mechanism of spontaneous combustion from a microscopic perspective. Wang [5] conducted adiabatic oxidation experiments to study the conversion of functional groups in coal samples of different ranks, and found that in the slow low-temperature accumulation process, alkyl side chains combine with oxygen atoms to form unstable intermediates, which then decomposed into gaseous products and stable solid complexes. Although the functional groups in low-rank coals have received extensive attention, there has no detailed research on those groups, which are abundant and play a major role in the process of ignition.

In this paper, two low-rank coals are selected, focusing on the changes of hydroxyl, carboxyl and alkyl side chain before ignition. The main reactions in line with the change trend are determined, and then compared and analyzed with the quantitative results of titration under the same temperature conditions. The heat release of low-rank coal before spontaneous combustion and the reaction enthalpy changes of the reactions are correlated to find the key temperature stage of sufficient heat storage which causes the spontaneous combustion of low-rank coal.

\section{Materials and Methods}

\subsection{Materials}

Table 1. Proximate and ultimate analyses of coal samples.

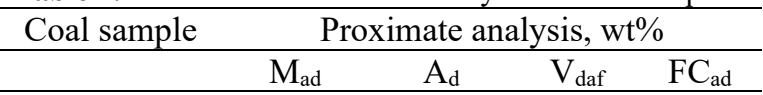




\begin{tabular}{cccccc}
\hline YJL52 & 2.36 & 10.08 & 35.39 & 58.1 \\
BRXL & 6.44 & 16.16 & 44.84 & 43.27 \\
\hline \multicolumn{7}{c}{ Ultimate analysis, $\mathrm{wt} \%$, daf } \\
\hline C & $\mathrm{H}$ & $\mathrm{O}^{\mathrm{a}}$ & $\mathrm{S}$ & $\mathrm{N}$ \\
YJL52 & 72.88 & 4.29 & 21.58 & 0.28 & 0.97 \\
BRXL & 58.46 & 3.81 & 36.82 & 0.23 & 0.68 \\
\hline
\end{tabular}

Note: ad, air-dried basis; d, dry basis; daf, dry and ash-free basis; a Calculated by difference.

Table 1 shows the results of proximate analysis and ultimate analysis of coal samples used in this article.

\subsection{Thermogravimetric experiment}

The pulverized low-rank coal samples $(10 \mathrm{mg})$ were subjected to TGA measurements ( NETZSCH, STA2500), in air atmosphere with the flow rate of 100 $\mathrm{mL} / \mathrm{min}$, the temperature range was set as $20-700{ }^{\circ} \mathrm{C}$ with a heating rate of $20 \mathrm{~K} / \mathrm{min}$, to determine the ignition temperature of the two coal samples. After that, in two different atmospheres (air, nitrogen) with $10 \mathrm{mg}$ samples for each treatment, heating BRXL from $20^{\circ} \mathrm{C}$ to $100^{\circ} \mathrm{C}$, $200{ }^{\circ} \mathrm{C}, 300{ }^{\circ} \mathrm{C}$, and $330{ }^{\circ} \mathrm{C}$, and heating YJL52 from $20{ }^{\circ} \mathrm{C}$ to $100{ }^{\circ} \mathrm{C}, 200{ }^{\circ} \mathrm{C}, 300{ }^{\circ} \mathrm{C}, 400{ }^{\circ} \mathrm{C}$ and $430{ }^{\circ} \mathrm{C}$. At the end of each heating treatment, the sample was cooled to room temperature in the thermogravimetry.

\subsection{Infrared spectroscopy experiment}

The FTIR spectra (IRTracer-100) were recorded in the interval between 400 and $4000 \mathrm{~cm}-1.1 \mathrm{mg}$ of the coal samples treated in section 2.2 was mixed with $150 \mathrm{mg}$ of spectroscopic grade $\mathrm{KBr}$. The obtained mixture was pressed to prepare the thin slices for measurements. Then the second derivative function of ORIGIN 9.0 was used to determine the position of the absorption peaks. Consequently, peak fitting was performed and the peak areas of alkyl side chains, hydroxyl and carboxyl at different temperature were obtained.

\subsection{Titration of carboxyl and phenolic hydroxyl on the coal surface}

The total acidity of phenolic hydroxyl and carboxyl on the surface of coal sample can be measured by the barium hydroxide solution, and cation exchange reaction was as follows[6]:

$$
2 \mathrm{HA}+\mathrm{Ba}(\mathrm{OH})_{2} \rightarrow \mathrm{BaA}_{2} \downarrow+2 \mathrm{H}_{2} \mathrm{O}
$$

The carboxyl content could be obtained by the calcium acetate solution, and the reaction was as follows: $\mathrm{OOH}$

$\mathrm{Ca}(\mathrm{CH} 3 \mathrm{COO}) 2+2 \mathrm{RCOOH} \rightarrow \mathrm{Ca}(\mathrm{RCOO}) 2 \downarrow+2 \mathrm{CH} 3 \mathrm{C}$

\section{Results and Discussion}

\subsection{The ignition temperature of YJL52 and BRXL}

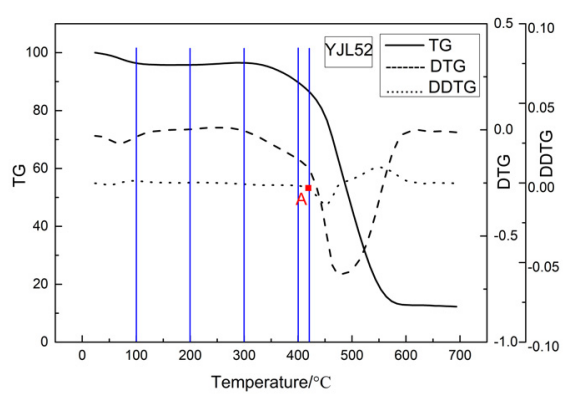

(a)

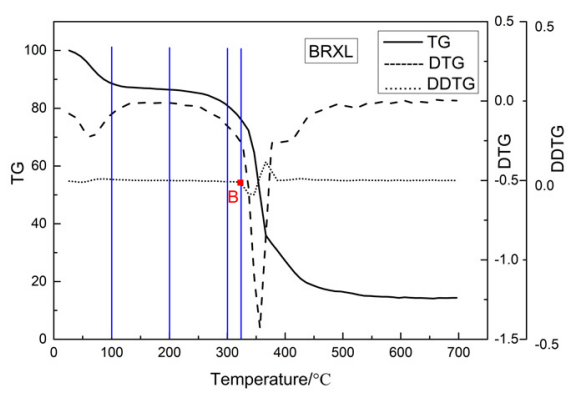

(b)

Fig. 1. The ignition temperature determination of YJL52 (a) and BRXL (b).

In Fig. 1, DDTG is the second derivative of the TG curve, representing the acceleration of mass loss. The point where the DDTG curve beginning to change from zero is the inflection point, which could indicate a sudden increase in the mass loss rate, at this time, the ignition temperature of the coal sample reached. Therefore, point $\mathrm{A}$ and point $\mathrm{B}$ represent the ignition temperature of YJL52 and BRXL, which is $430{ }^{\circ} \mathrm{C}$ and $330{ }^{\circ} \mathrm{C}$, respectively.

\subsection{The change of alkyl side chain, hydroxyl and carboxyl}

As shown in Fig. 2(a), at the stage of $20-100{ }^{\circ} \mathrm{C}$, three functional groups of YJL52 slightly decrease in nitrogen atmosphere and air atmosphere. It suggests that there are reactions happened related to these functional groups below $100{ }^{\circ} \mathrm{C}$, which are similar with the research results in the Ref. [7]. In air atmosphere, the alkyl side chains are less than that in nitrogen atmosphere, which means that the coal-oxygen reaction consumes the alkyl side chains [8]. At the stage of $100-200{ }^{\circ} \mathrm{C}$, the alkyl side chains begin to increase greatly in nitrogen atmosphere and air atmosphere. Similarly, in air atmosphere, the alkyl side chains are less than that in nitrogen atmosphere, indicating that the alkyl side chain also oxidizes at this stage. In two kinds of atmospheres, the changes of hydroxyl and carboxyl are almost same, meaning that there is little oxidation process related with these two groups. At the stage of $200-300{ }^{\circ} \mathrm{C}$, in air atmosphere, alkyl side chains decrease, while in nitrogen atmosphere, alkyl side chains are still increasing. At the same time, in air atmosphere, hydroxyl and carboxyl significantly increase. In nitrogen atmosphere, hydroxyl and carboxyl continuously decrease. With the temperature increasing, the influence of oxygen on these 
groups is gradually obvious, and the opposite trend also implies that alkyl side chain might be converted to the hydroxyl and carboxyl by oxidation reaction. At the stage of $300-400{ }^{\circ} \mathrm{C}$, in the air atmosphere, alkyl side chains continue to decrease, while in nitrogen atmosphere, alkyl side chains are still increasing. Meanwhile, in air atmosphere, hydroxyl and carboxyl also appear an obvious decrease, which demonstrates that at this temperature interval, the conversion from alkyl side chain to hydroxyl and carboxyl is still taking place, but the decomposition and conversion between hydroxyl and carboxyl begin to dominate. At the stage of $400-430{ }^{\circ} \mathrm{C}$, three groups all reduce, especially hydroxyl and carboxyl, which are about to be exhausted.

For BRXL, as shown in Fig. 2(b), in air atmosphere, the trend is similar to YJL52, which suggests that although different low-rank coals have significant differences in composition and ignition temperature, the reactions occur before ignition might be in common. At the stage of $200-300{ }^{\circ} \mathrm{C}$, in nitrogen atmosphere, hydroxyl groups greatly increase, while carboxyl and alkyl side chains appear a decrease. Cui [9] believed that the formation of hydroxyl under pyrolysis conditions is mainly caused by the free hydrogen generated by the thermal decomposition of the other oxygen-containing functional groups and the oxygen generated by the thermal decomposition. The proximate analysis results of the two coals show that the fixed carbon content of BRXL is very low, which means that it may contain more other oxygen-containing functional groups and the conversion reactions to hydroxyl are prone to occur, causing the increase of hydroxyl in nitrogen atmosphere.

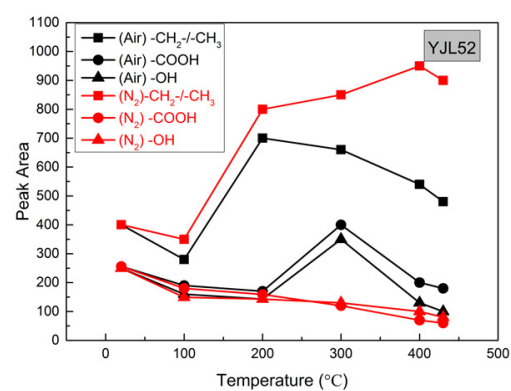

(a)

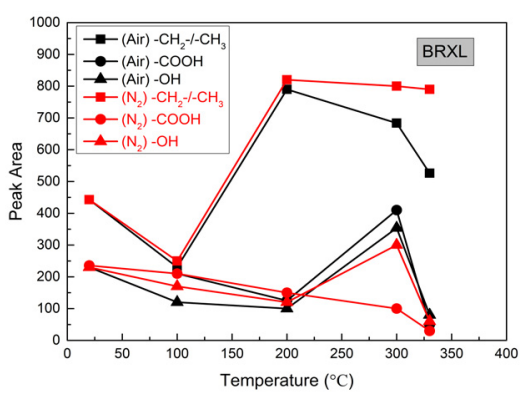

(b)

Fig. 2. The change of alkyl side chain, hydroxyl and carboxyl of YJL52 (a) and BRXL (b) in two atmospheres.

Based on the analysis of the change of alkyl side chain, hydroxyl and carboxyl at different temperature stages, it could be speculated that oxidization of alkyl side chains to hydroxyl and carboxyl, and decomposition of carboxyl mainly happen before the ignition.

\subsection{Reactions analysis}

According to the Ref. [10], the most active group is $\mathrm{R} 3-\mathrm{CH}$ in low-rank coal. It first spontaneously forms $\mathrm{R} 3-\mathrm{C} \cdot$ and $\mathrm{HOO}$ - under the attack of oxygen. Then hydrogen peroxide radicals form hydroxyl radicals, which can induce other alkyl groups to generate free radicals. The above processes are all spontaneous reactions, which can be expressed by formula (1).

$$
\mathrm{R}_{3}-\mathrm{CH} \underset{\text { Spontaneous }}{\stackrel{\mathrm{O}_{2}}{\longrightarrow}} \mathrm{R}_{3} \mathrm{C} \cdot+\stackrel{\dot{\mathrm{O}}-\mathrm{O}-\mathrm{H}}{\downarrow}+\mathrm{R}-\mathrm{H} \underset{\text { Spontaneous }}{\longrightarrow} \mathrm{R}
$$

The formula (2) presents the conversion process from the alkyl side chain to the hydroxyl and carboxyl. The alkyl radicals formed in the formula (1) spontaneously form peroxyl radicals ROO', and then peroxyl radicals generate alkoxy radicals $\mathrm{R}-\mathrm{CH}-\mathrm{O} \cdot$. The conversion to $\mathrm{R}-\mathrm{CH}-\mathrm{O} \cdot$ is not spontaneous, which needs to be initiated at a higher temperature. After that, alkoxy radicals combine with hydrogen radicals to form hydroxyl groups. In addition, R-CH-O could generate aldehyde which then turns into aldehyde radicals. Under the effect of oxygen, aldehyde spontaneously forms carboxyl radicals which combine with hydrogen radicals to form carboxyl. Only a non-spontaneous reaction is in this process, therefore, it does not require very high temperature to occur.

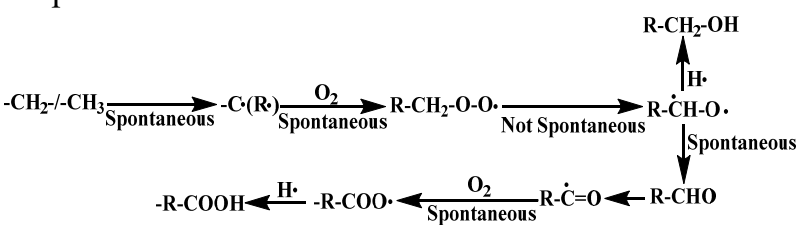

Formula (3) presents the process of conversion of hydroxyl to carboxyl. The hydroxyl spontaneously forms alkoxy radicals which then turn into aldehyde groups, and aldehyde groups generate carbonyl radicals, carboxyl radicals could be formed spontaneously under the action of oxygen. The carboxyl radicals combine with hydrogen radicals to form carboxyl groups. This process is a spontaneous reaction and could occur at a low temperature.

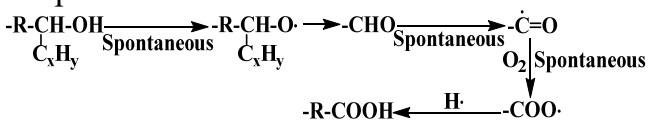

Formula (4) presents the process of decomposition of carboxyl groups to form alkyl side chains. Carboxyl groups spontaneously generate carboxyl radicals, carboxyl radicals don't spontaneously generate alkyl radicals, and alkyl radicals combine with hydrogen radicals to form alkyl side chains. This process is very easy to generate carboxyl radicals, but the decarboxylation of carboxyl radicals to generate alkyl radicals requires a slightly higher temperature. $-\mathrm{R}-\mathrm{COOH} \underset{\text { Spontaneous }}{\longrightarrow}-\mathrm{R}-\mathrm{COO} \cdot \underset{\text { Not Spontaneous }}{\longrightarrow}-\mathrm{R}+\mathrm{CO}_{2} \stackrel{\mathrm{H} \cdot}{\longrightarrow}-\mathrm{R}$

Based on the above analysis, the main relevant reactions could be reasonably determined, which are as 
follows: the oxidation of alkyl side chain to hydroxyl, the oxidation of hydroxyl to carboxyl, and the decarboxylation of carboxyl to alkyl side chain.

\subsection{The titration of phenolic hydroxyl and carboxyl on the surface of coal}

The proximate analysis of coal samples displays that YJL52 has a high volatile content, while BRXL has a very low content of fixed carbon, but its volatile content, water content and ash content are high. The component characteristics are also reflected by the difference of original carboxyl and hydroxyl of the two coal samples. The amount of phenolic hydroxyl of two coals is similar, YJL52 is slightly higher, but the amount of carboxyl in BRXL is significantly more than that in YJL52. The ignition temperature of BXRL is about $100{ }^{\circ} \mathrm{C}$ lower than that of YJL52, which means that the reaction exotherm related with surface carboxyl may have a certain effect on the easier ignition of BXRL. Although the ignition temperature is different, the variation tendency of the phenolic hydroxyl and carboxyl on the surface of the two coals is little difference below $300{ }^{\circ} \mathrm{C}$, which suggests that the phenolic hydroxyl and carboxyl on the surface have consistent effects on the ignition.

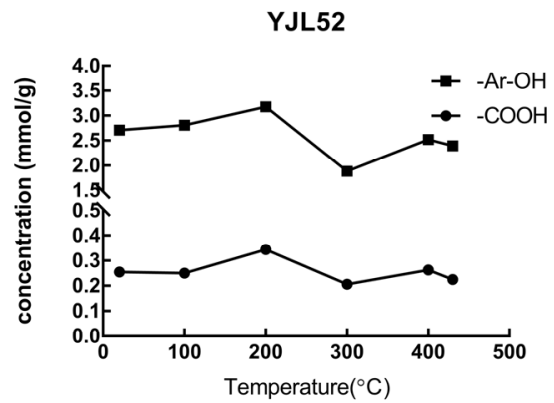

(a)

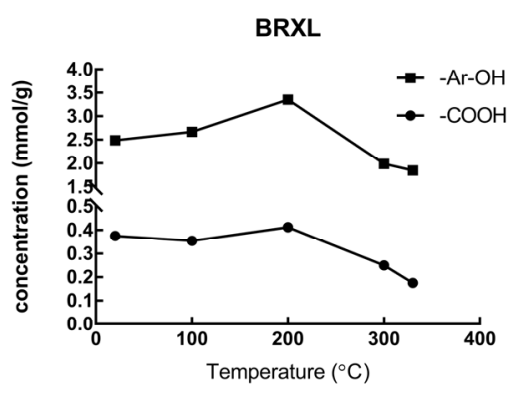

(b)

Fig. 3. Titration results of phenolic hydroxyl and carboxyl groups on the surface of YJL52 (a) and BRXL (b).

As shown in Fig.3, at the stage of $20-100{ }^{\circ} \mathrm{C}$,

Table 2. Comparison of infrared measurement and titration results.

\begin{tabular}{|c|c|c|c|c|c|c|c|}
\hline coal & method & functional group & $20-100^{\circ} \mathrm{C}$ & $100-200^{\circ} \mathrm{C}$ & $200-300^{\circ} \mathrm{C}$ & $300-330^{\circ} \mathrm{C}$ & \\
\hline \multirow{4}{*}{ BRXL } & Infrared & Total hydroxyl & $\downarrow$ & $\downarrow$ & $\uparrow$ & $\downarrow$ & \\
\hline & spectroscopy & Total carboxyl & $\downarrow$ & $\downarrow$ & $\uparrow$ & $\downarrow$ & \\
\hline & Titration & Phenolic hydroxyl & $\uparrow$ & $\uparrow$ & $\downarrow$ & $\downarrow$ & \\
\hline & litration & Carboxyl & $\downarrow$ & $\uparrow$ & $\downarrow$ & $\downarrow$ & \\
\hline & & & $20-100^{\circ} \mathrm{C}$ & $100-200^{\circ} \mathrm{C}$ & $200-300^{\circ} \mathrm{C}$ & $300-400^{\circ} \mathrm{C}$ & $400-430^{\circ} \mathrm{C}$ \\
\hline \multirow{4}{*}{ YJL52 } & Infrared & Total hydroxyl & $\downarrow$ & $\downarrow$ & $\uparrow$ & $\downarrow$ & $\downarrow$ \\
\hline & spectroscopy & Total carboxyl & $\downarrow$ & $\downarrow$ & $\uparrow$ & $\downarrow$ & $\downarrow$ \\
\hline & Titration & Phenolic hydroxyl & $\uparrow$ & $\uparrow$ & $\downarrow$ & $\uparrow$ & $\downarrow$ \\
\hline & & Carboxyl & $\downarrow$ & $\uparrow$ & $\downarrow$ & $\uparrow$ & $\downarrow$ \\
\hline
\end{tabular}

phenolic hydroxyl slightly increases and carboxyl slightly decreases, which demonstrates that there may be reactions generating phenolic hydroxyl and carboxyl decomposition. At the stage of $100-200{ }^{\circ} \mathrm{C}$, phenolic hydroxyl and carboxyl increase, it can be concluded that with the temperature increases, the oxidation of the groups gradually dominates. At the stage of $200-300{ }^{\circ} \mathrm{C}$, both phenolic hydroxyl and carboxyl significantly reduce. When the temperature increases by $30{ }^{\circ} \mathrm{C}$ and reaches $330{ }^{\circ} \mathrm{C}$, BRXL ignites. This stage closes to the temperature of ignition, hence, the reactions that consume the two acidic groups at this stage have a significant effect on spontaneous combustion. For BRXL, at the stage of $300-330{ }^{\circ} \mathrm{C}$, the phenolic hydroxyl and carboxyl continue to decrease, and the reactions that consume the similar groups further promote the ignition. For YJL52, there is a small increase in the two groups at the stage of $300-400{ }^{\circ} \mathrm{C}$, which may be caused by the continuous occurrence of oxidation reaction. Similarly, when the temperature reaches $430{ }^{\circ} \mathrm{C}$, the phenolic hydroxyl and carboxyl both reduce a little, suggesting the groups consumption further releases heat and leads to ignition eventually.

\subsection{Effect of the temperature stage on the ignition of low-rank coals}

\subsubsection{The main reaction at each temperature stage}

Table 2 shows the comparison results obtained by IR and titration of the hydroxyl and carboxyl at each temperature stage before the ignition. It is obvious that to BRXL and YJL52, the variation tendencies of the two groups are completely consistent when the temperature is

At the stage of $20-100{ }^{\circ} \mathrm{C}$, the total hydroxyl and total carboxyl decrease, but the phenolic hydroxyl groups increase, indicating that a small amount of phenolic hydroxyl are generated, meanwhile, more alcoholic hydroxyl groups are oxidized to form carboxyl. While the total carboxyl groups decrease at this time, suggesting that carboxyl undergoes decomposition at this stage. As a result, the consumption of carboxyl is greater than the amount produced, that is, the main reaction at this stage is the decomposition reaction of carboxyl. At the stage of $100-200{ }^{\circ} \mathrm{C}$, the total hydroxyl and total carboxyl decrease, but the surface phenolic hydroxyl and surface carboxyl increase, implying that the reaction to below $300^{\circ} \mathrm{C}$. 


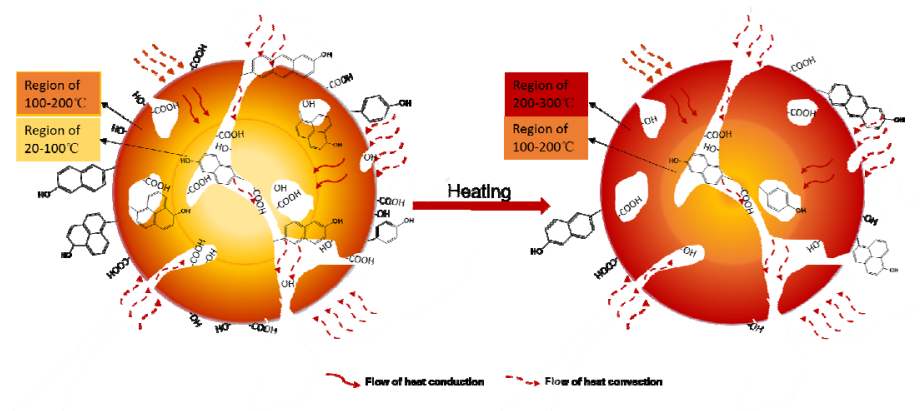

Fig. 4. Schematic diagram of heat transfer lag inside and outside BRXL coal particle.

generate phenolic hydroxyl is still proceeding on the surface of coal particles. At the same time, more alcoholic hydroxyl groups are oxidized to form carboxyl on the surface, leading to the total hydroxyl groups decrease. The change of surface carboxyl and total carboxyl are opposite, which may be caused by the delay of heat transfer between inside and outside the coal particles. As shown in Fig. 4(a), when the temperature of surface reaches $100-200{ }^{\circ} \mathrm{C}$, there will be heat loss in the heat conduction process [11], causing the temperature inside the coal particles lower than its surface temperature which may still be at the temperature of 20-100 ${ }^{\circ} \mathrm{C}$. At this temperature, the carboxyl decomposition is the main reaction. Therefore, although the surface carboxyl groups increase due to the oxidation of alcoholic hydroxyl, the total carboxyl groups decrease due to the internal carboxyl decomposition. At the stage of $200-300{ }^{\circ} \mathrm{C}$, the total hydroxyl and total carboxyl increase, and the surface phenolic hydroxyl and surface carboxyl decrease, indicating that the phenolic hydroxyl groups on the surface are converted into alcoholic hydroxyl, and a large number of alkyl side chains are oxidized to produce alcoholic hydroxyl on the surface, causing increase in total hydroxyl groups. The delay of heat transfer between inside and outside coal particles also exists at this temperature stage, and it also causes the change of surface carboxyl and total carboxyl to be opposite. As shown in Fig. 4(b), the inside coal is still at 100-200 ${ }^{\circ} \mathrm{C}$, at this temperature stage, the main reaction is the oxidation of alcoholic hydroxyl to form carboxyl, so the carboxyl inside coal increase. Although the surface carboxyl decrease, the total carboxyl groups increase. When the temperature is higher than $300{ }^{\circ} \mathrm{C}$, BRXL is close to ignition. At the stage of $300-330{ }^{\circ} \mathrm{C}$, the total carboxyl, total hydroxyl, surface phenolic hydroxyl and surface carboxyl demonstrate a decrease. The main reaction at this stage may be the decomposition reaction of carboxyl groups, which consumes the carboxyl and then hydroxyl, causing simultaneous reduction of carboxyl and hydroxyl.

For YJL52, the change at the stage of $300-400{ }^{\circ} \mathrm{C}$ is the same as that at the stage of $100-200{ }^{\circ} \mathrm{C}$, it is speculated that the main reactions on the surface and inside coal are also identical with that at the $100-200{ }^{\circ} \mathrm{C}$ stage. At the stage of $400-430{ }^{\circ} \mathrm{C}$, the reason for the reduction of groups is the same as above analyses of the approaching ignition of BRXL.

\subsubsection{Heat analysis of each temperature stage}

As shown in Table 3, according to the Refs. [12,13], the length of side chain of BRXL and YJL52 could be estimated as 7 and 5, and then the quantum chemical method can be used to calculate the enthalpy changes of the oxidation of alkyl side chain to hydroxyl, the oxidation of hydroxyl to carboxyl, and the decarboxylation of carboxyl to alkyl side chain. In addition, the heat release of each stage is calculated based on the DTA curve and the results are exhibited in Table 4.

The data in Table 4 shows that at the stage of 20-100 ${ }^{\circ} \mathrm{C}$, although the decomposition of the carboxyl groups is the main reaction, this stage is endothermic, which is due to the physical evaporation of water in coal. At the stage of $100-200{ }^{\circ} \mathrm{C}$, the main reaction is the oxidation of alcoholic hydroxyl groups to carboxyl groups. It can be seen from Table 3 that the reaction enthalpy change of the reaction is as high as $361 \mathrm{~kJ} / \mathrm{mol}$, the exothermic heat of this stage is very low, which illustrates that at this stage, the number of functional groups involved in the reaction still less. At the stage of 200-300 ${ }^{\circ} \mathrm{C}$, the main reaction enthalpy change of the alkyl side chain oxidation is $215 \mathrm{~kJ} / \mathrm{mol}$, but the total heat release of the two coal samples at this stage is much higher than that of other temperature stages, which suggests that there are a lot of oxidation reactions of the alkyl side chain take place, which make this temperature section a key stage that causes the low-rank coal to ignite. At the stage of $300-330{ }^{\circ} \mathrm{C}$, the exothermic heat of BRXL decreases sharply to only $1.5 \mathrm{~J} / \mathrm{g}$, indicating that at this stage, most of the main functional groups in the coal have been consumed, and there is almost no effect on the ignition.

For YJL52, although the exotherm at the stage of $300-400{ }^{\circ} \mathrm{C}$ is reduced to $78 \mathrm{~J} / \mathrm{g}$, it is still another important stage, this may be because the number of

Table 3. Typical reactions and their enthalpy changes $(\Delta H)$.

\begin{tabular}{ccc} 
Coal & Reaction & $\Delta H(\mathrm{~kJ} / \mathrm{mol})$ \\
\hline $\mathrm{BRXL}$ & & \\
\hline
\end{tabular}




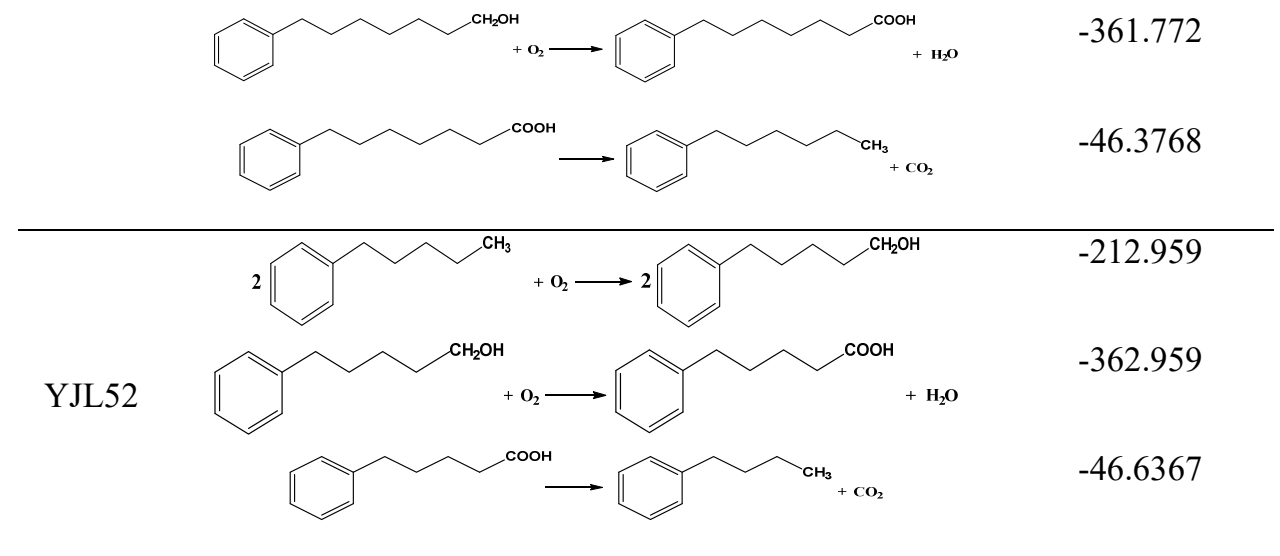

Table 4. Heat absorption and heat release of each temperature stage.

\begin{tabular}{cccccc}
\hline Coal sample & \multicolumn{5}{c}{ BRXL } \\
\hline Temperature interval $\left({ }^{\circ} \mathrm{C}\right)$ & $20-100$ & $100-200$ & $200-300$ & $300-330$ \\
Quantity of heat $(\mathrm{J} / \mathrm{g})$ & 43.55349 & -4.74187 & -167.755 & -1.50474 \\
\hline Coal sample & & YJL52 & \\
\hline Temperature interval $\left({ }^{\circ} \mathrm{C}\right)$ & $20-100$ & $100-200$ & $200-300$ & $300-400$ & $400-430$ \\
Quantity of heat $(\mathrm{J} / \mathrm{g})$ & 36.19993 & -6.51999 & -155.08291 & -78.3518 & -7.68658 \\
\hline
\end{tabular}

original groups in YJL52 is slightly less than that in BRXL. As a result, at the previous stage, the heat storage is not enough to cause ignition, and then the rest of groups continue to react and release more heat with the temperature increase. At the stage of $400-430^{\circ} \mathrm{C}$, the heat released also sharply decreases to $7.68 \mathrm{~J} / \mathrm{g}$, which is similar to the stage prior to the ignition of BRXL, and it is also no effect on the ignition.

\section{Conclusions}

The changes of hydroxyl, carboxyl, and alkyl side chains at different temperature stages before ignition of two coals are studied by IR. Further variation of the phenolic hydroxyl and carboxyl on coal surface are detected by titration. It can be inferred that the main reaction at $20-100{ }^{\circ} \mathrm{C}$ is the decomposition reaction of carboxyl. When the temperature is higher than $100{ }^{\circ} \mathrm{C}$, the rate of heat transfer exhibits difference inside and outside coal samples. The main reaction on the surface at $100-200{ }^{\circ} \mathrm{C}$ is the oxidation of alcoholic hydroxyl to carboxyl groups, the main reaction at $20-100{ }^{\circ} \mathrm{C}$ still occurs inside the coal. The main reaction at $200-300{ }^{\circ} \mathrm{C}$ is conversion of phenolic hydroxyl to alcoholic hydroxyl, oxidization of alkyl side chain to alcoholic hydroxyl and decomposition of carboxyl on surface. The main reaction at $100-200{ }^{\circ} \mathrm{C}$ occurs inside the coal sample. The oxidation of alcoholic hydroxyl to carboxyl group mainly happens on the surface at $300-400{ }^{\circ} \mathrm{C}$, the reaction inside is same as the main reaction at $200-300{ }^{\circ} \mathrm{C}$. In addition, combining the heat release in each temperature stage with the enthalpy change of the main reaction, it is found that although the composition of the two coals is quite different, the stage of $200-300{ }^{\circ} \mathrm{C}$ is the main period of heat storage. At this stage, there are a large number of conversion reactions of functional groups occur.

In a word, the main reaction of the main functional groups and the effect of each temperature stage on the ignition are revealed, which further improves the reaction mechanism of low-rank coal spontaneous combustion.

\section{Acknowledgments}

This work was supported by grants from the National Natural Science Foundation of China [Grant No. 21606083, and the Fundamental Research Funds for the Central Universities [Grant No, 2016MS017].

\section{References}

1. D.G. Lee, Y.Y. Isworo, K.H. Park, G.M. Kim, S.M. Kim, ACS. Omega 5, 18594 (2020)

2. A. Arisoy, B. Beamish, Fuel 159, 412 (2015)

3. X. Zhai, B. Wang, K. Wang, D Obracaj, Combust. Sci. Technol 191, 1101 (2019)

4. Y. Zhang, Y. Li, Y. Huang, S. Li, W. Wang, J. Therm. Anal. Calorim 131, 2963 (2018)

5. D. Wang, X. Zhong, J. Gu, X. Qi, Min. Sci. Technol 20, 35 (2010)

6. H.N.S. Schafer, Fuel 63, 723 (1984)

7. Z. D, Y. Z, Coal Conversion 01, 54 (1997)

8. H. Wang, B.Z. Dlugogorski, E.M. Kennedy, Combust. Flame 134, 107 (2003)

9. X. Cui, X. Li, Y. Li, S. Li, J. Therm. Anal. Calorim 129, 1169 (2017)

10. D. Wang, H. Xin, X. Qi, G. Dou, G. Qi, L. Ma, Combust. Flame 163, 447 (2016)

11. X. Liu, G. Wang, G. Pan, Z. Wen, Fuel 106, 667 (2013)

12. X. He, X. Liu, B. Nie, D. Song, Fuel 206, 555 
(2017)

13. F. Yang, Y. Hou, W. Wu, Z. Liu, Energ. Fuel 31, 11 (2017) 